\title{
二甲双胍硫化氢盐的合成、结构鉴定和稳定性研究
}

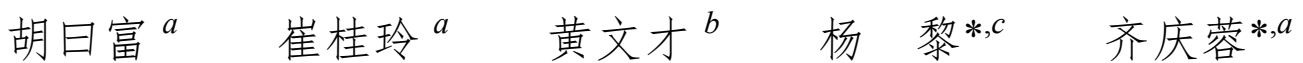 \\ $\left({ }^{a}\right.$ 四川大学华西药学院 靶向药物与释药系统教育部重点实验室 四川省植物来源药物工程实验室 \\ 四川省药物精密工业技术研究中心 成都 610041) \\ ( ${ }^{b}$ 四川大学化学工程学院 成都 610065) \\ ( ${ }^{c}$ 四川大学华西医院肿瘤中心 成都 610041)
}

\begin{abstract}
摘要 随着硫化氢作为人体内重要内源性气体信号分子研究的不断深入, 有机小分子硫化氢供体的研究也取得了较大 的进展，但目前尚无一种硫化氢供体药物上市.二甲双胍是临床上治疗糖尿病的一线药物，近年来被报道还具有减肥、 抗癌、抗衰老等多种生理活性. 鉴于硫化氢和二甲双胍在重大疾病治疗中的良好前景, 设计了一种全新的硫化氢供体 二甲双胍硫化氢盐，前人对该类有机碱硫化氢盐的系统研究几乎为空白，更无将其作为硫化氢供体的报道. 首先将二 甲双胍盐酸盐处理得到二甲双胍游离碱，再与硫化氢反应形成二甲双胍硫化氢盐. 通过氢谱、碳谱、元素分析及 $\mathrm{X}$ 单 晶衍射对其进行了结构确证. 采用碘量法、醋酸铅法和苂光亚甲蓝法测定了二甲双胍硫化氢盐中硫化氢的含量，均大 于 $96.00 \%$; 采用高效液相色谱(HPLC)测定了二甲双胍硫化氢盐中二甲双胍的含量大于 $98.50 \%$ ，且二甲双胍硫化氢盐 的溶液及固体均相对稳定. 上述研究结果表明, 二甲双胍硫化氢盐有望作为一种新结构类型的硫化氢供体进行深入的 研究和开发.
\end{abstract}

关键词 二甲双胍硫化氢盐; 硫化氢供体; 硫化氢治疗

\section{Synthesis, Structural Characterization and Stability Evaluation of Metformin Hydrosulfide}

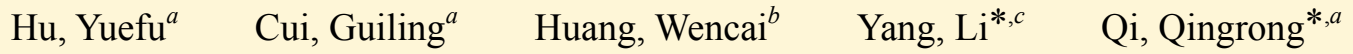 \\ $\left({ }^{a}\right.$ Key Laboratory of Drug-Targeting and Drug Delivery System of the Education Ministry, Sichuan Engineering Laboratory \\ for Plant-Sourced Drug and Sichuan Research Center of Drug Precision Industrial Technology for \\ Small Molecule Drugs, West China School of Pharmacy, Sichuan University, Chengdu 610041) \\ ( ${ }^{b}$ School of Chemical Engineering, Sichuan University, Chengdu 610065) \\ ( ${ }^{c}$ Cancer Center, West China Hospital, Sichuan University, Chengdu 610041)
}

\begin{abstract}
Hydrogen sulfide $\left(\mathrm{H}_{2} \mathrm{~S}\right)$, the third gas signal molecule in mammals is studied more and more deeply, and great progress has been made on organic small molecule hydrogen sulfide donors. However, no hydrogen sulfide donor has been marketed as a drug yet. Metformin is a first-line drug for the treatment of diabetes. And some other biological activities such as anti-obesity, anticancer, and anti-aging have been disclosed in recent years. $\mathrm{H}_{2} \mathrm{~S}$ donor has also shown promising prospect in antitumor, cardiovascular protection, anti-inflammation, ion channel regulation and anti-oxidation activities. In view of the potential of hydrogen sulfide and metformin for treatment of diseases, a novel hydrogen sulfide donor, metformin hydrosulfide, was thus designed. Till now, there is no report of this type of hydrogen sulfide donor. In our work, metformin hydrochloride was first basified to obtain metformin, followed by reaction with hydrogen sulfide to afford metformin hydrosulfide. The structure was fully characterized by ${ }^{1} \mathrm{H} N M R,{ }^{13} \mathrm{C}$ NMR, elemental analysis and X-ray crystal. The content of hydrogen sulfide was determined by iodometry method, lead acetate test and methylene blue spectrophotometric method to be more than $96.00 \%$. The metformin level was determined by high-pressure liquid chromatography (HPLC) to be more than $98.50 \%$. The stability of metformin hydrosulfide was evaluated by iodometry method in solution and solid, respectively. The results indicate that metformin hydrosulfide is a promising new type of hydrogen sulfide donor which deserves further research and development.
\end{abstract}

Keywords metformin hydrosulfide; hydrogen sulfide donor; hydrogen sulfide-based therapeutics

* Corresponding authors. E-mail: yangli@scu.edu.cn; qiqingrong@scu.edu.cn

Received November 26, 2018; revised December 26, 2018; published online January 8, 2019.

Project supported by the Construction of a Comprehensive Platform for the Innovation of Cardio-cerebrovascular Drugs in Shijiazhuang Pharmaceutical Group Company Limited (No. 2013ZX09402103-1).

石药集团心脑血管药物创新综合平台建设基金(No. 2013ZX09402103-1)资助项目. 
硫化氢 $\left(\mathrm{H}_{2} \mathrm{~S}\right)$ 因其恶臭、高毒，曾被列为有毒气体， 被认为毫无医药用途. 但近年来通过对其在体内生物 系统中影响生理病理过程的了解, 发现 $\mathrm{H}_{2} \mathrm{~S}$ 作为继 $\mathrm{NO}$ 和 $\mathrm{CO}$ 以后的第三种气体信号分子, 具有广泛的生物 学效应 ${ }^{[1]}$, 在多种重大疾病的治疗中显示了良好的前 景. 内源性硫化氢可通过胱硫醚- $\gamma$-裂解酶 (CSE)、胱硫 醚- $\beta$-合成酶 (CBS) 以及 3 -颈基丙酮酸硫基转移酶 (3-MST)产生 ${ }^{[2]}$. 目前, 内源性硫化氢已被证实, 在心 血管、神经、呼吸、消化、血液、新陈代谢等多系统 中具有重要调节作用 ${ }^{[2 \mathrm{a}, 3]}$.

除内源性硫化氢的生物调控作用外, 由硫化氢供体 释放的外源性硫化氢还可能起治疗作用, 目前报道的硫 化氢供体主要有两类. 第一类为无机 $\mathrm{H}_{2} \mathrm{~S}$ 供体, 如 $\mathrm{Na}_{2} \mathrm{~S} 、 \mathrm{NaHS}$, 这类供体在机体内释放明确, 无明显副产 物, 在糖尿病、神经保护等研究中表现出有意义的结果. 但无机硫化氢供体存在一些待解决的问题, 如需实现高 效精准的检测、水溶液的稳定性不佳等. 第二类为有机 $\mathrm{H}_{2} \mathrm{~S}$ 供体, 根据其释放硫化氢方式的不同, 有机硫化氢 供体主要有包括如下几种: (1)通过游离硫醇活化机理释 放硫化氢, 此类供体如大蒜中含有的已二烯二硫化物 (DADS) 1、已二烯三硫化物(DATS)等 ${ }^{[4]}$, 硫酰胺类 $\mathbf{2}^{[5]}$, 异硫氰酸酯类 $\mathbf{3}^{[6]}, N$-硫兼基取代衍生物(NSHD) $4^{[7]}$, 过 硫化合物(perthiol) $5^{[1]]}$ 等; (2)通过水解反应释放硫化氢, 此类供体如 Lawesson's 试剂 $\mathbf{6}^{[8]} 、 1,2-$ 二硫-3-硫酮衍生 物(DTTS) 类 ${ }^{[9]}$ 及 1,2-二硫-3-硫酮(DTT)和非甾体抗炎药 (NSAIDs) 如双氯芬酸钠等杂合的衍生物 $7^{[10]}$ GYY4317 $8^{[2 \mathrm{a}, 11]}$ 等; (3)紫外光催化释放硫化氢, 此类供体如偕二 硫醇 $\mathbf{9}^{[12]}$ 、酮取代的布洛芬衍生物 $\mathbf{1 0} \mathbf{0}^{[13]}$ 、氧杂葱酮类 衍生物 ${ }^{[14]}$ 等; (4)通过 $\mathrm{pH}$ 调控硫化氢的释放, 此类供体 如硫代氨基酸 $\mathbf{1 1}^{[15]}$; (5)通过酯酶催化释放硫化氢, 此类 供体如酯酶敏感型类化合物 $\mathbf{1 2}^{[16]}$; (6)通过碳酸䣲酶水 解释放硫化氢, 此类供体如硫羰基类化合物 $\mathbf{1 3}^{[17]}$.

上述有机硫化供体在生物学评价时, 大部分都表现 出了良好的生物活性，但也存在一些问题 ${ }^{[1]}$ : (1)大多供 体释放硫化氢的速度不可控; (2)有些供体如 DTT 类、劳 森试剂等, 供体本身水溶性很差, 且具有恶臭; (3)有些 供体如 GYY4137 本身具有活性, 且释放硫化氢的机理 只是推测, 并未真正阐明, 所以难以准确判断其表现出 来的生物活性是供体本身还是硫化氢产生的; (4)部分供 体释放硫化氢的条件如紫外催化和 $\mathrm{pH}$ 调控, 在生物体 实现的可行性不高, 如通过紫外光敏的硫化氢供体, 其 细胞毒性和有限的光穿透能力使其应用受到了一定的 限制; (5)很多情况下, 为了达到硫化氢发挥生物效应的 微摩尔浓度, 需要毫摩尔的有机供体, 如此高浓度的底 物可能带来许多其他问题, 如毒性、溶解性以及和生物<smiles>[R]C(=O)NSC([Z])=O</smiles><smiles>COc1ccc(P(=S)(S)N2CCOCC2)cc1</smiles><smiles>[R]C([R])(SCc1ccccc1[N+](=O)[O-])SCc1ccccc1[N+](=O)[O-]</smiles><smiles>[R]C(N)C(=O)S</smiles><smiles>[R]C(=O)Oc1cccc([R2])c1C(C)(C)CC(N)=O</smiles><smiles>N#Cc1ccc(COC(=S)Nc2ccc(F)cc2)cc1</smiles>

体其他组分的相互作用; (6)有机硫化氢供体在制备和保 存时的化学稳定性也需要高度关注. 所以, 开发新型硫 化氢供体具有重要的理论价值和应用前景.

目前国际上硫化氢供体的研究思路还是设计新型 有机小分子硫化氢供体, 核心在于可控地释放硫化氢但 尚未有药物上市, 也未曾提出用有机碱的硫化氢盐作为 硫化氢供体, 用于重大疾病的治疗.

除本课题组专利 ${ }^{[18]}$ 报道的 15 个有机碱硫化氢盐以 外, 文献报道的有机碱硫化氢盐多为简单胺类化合物, 且未见有机碱硫化氢盐相关化学的系统研究, 亦无有机 碱硫化氢盐的生物活性报道. 1931 年 Achterhof 等 ${ }^{[19]}$ 报 道了 12 个简单有机碱硫化氢盐的合成方法以及它们的 熔点、元素分析和颜色性状等理化性质. 其它一些有机 碱硫化氢盐多是作为反应副产物被报道, 而硫化氢作为 重要的内源性活性物质, 是最近 20 多年来才被发现, 因 此有机碱硫化氢盐是药物化学中尚未开发的化合物类 型.

二甲双胍是临床上广泛应用的治疗糖尿病的小分 子药物，近年来被报道还具有减肥、抗衰老等多种生理 活性 ${ }^{[20]}$, 同时也是一种碱性很强的有机碱, 而硫化氢是 一种弱酸性物质，故本文设想利用酸碱成盐原理，将市 售的二甲双胍盐酸盐处理成强碱性的二甲双胍，再与硫 化氢成盐，制成二甲双胍的硫化氢盐(DMGS).二甲双 胍硫化氢盐可作为第三种创新类型的硫化氢供体, 且二 
甲双胍是治疗糖尿病的一线用药, 其有效性和安全性得 到了多年的临床验证. 鉴于此, 本文合成了二甲双胍硫 化氢盐, 并通过氢谱、碳谱、元素分析及 $\mathrm{X}$ 单晶衍射进 行了结构鉴定, 通过碘量法、醋酸铅法和荧光亚甲蓝法 测定了硫化氢的含量, 并通过 HPLC 测定了二甲双胍的 含量. 二甲双胍硫化氢的溶液稳定性及固体稳定性结果 表明, 该化合物有望作为一种新型小分子硫化氢供体. 考虑到二甲双胍硫化氢盐良好的应用前景, 其药理学研 究和其他有机碱硫化氢盐的系统化学研究正在进行中.

$$
\text { 1,1-二甲基双胍硫化氢盐(DMGS) }
$$

\section{1 结果与讨论}

\section{1 目标化合物的合成}

市售二甲双胍是 1,1 -二甲基双胍盐酸盐，合成二甲 双胍硫化氢盐需要首先得到强碱性的二甲双胍游离碱, 再与弱酸性的硫化氢成盐(Scheme 1). 本文参考文献 [21]方法, 在笁选了一系列将二甲双胍盐酸盐游离的条 件后, 发现在金属钠/乙醇体系的条件下可以得到高纯 度的二甲双胍游离碱, 并获得了单晶结构, 数据详见结 构鉴定部分.

$$
\begin{aligned}
& \text { EtOH, r.t. } \\
& \text { DMG }
\end{aligned}
$$

图式 1 目标化合物 DMGS 的合成路线

Scheme 1 Synthetic route of target compounds DMGS

后续研究发现, 游离二甲双胍的纯度对于二甲双胍 硫化氢盐的质量具有决定性影响, 本文参考文献[21]采 用金属钠/乙醇体系得到二甲双胍游离碱粗品, 进而以 丙酮为溶剂反复脱盐析晶, 得到高纯度的二甲双胍晶 体. 相较于其它文献[22]报道, 如采用氢氧化钠为碱进 行游离, 该体系所得产物纯度高, 炽灼残渣显示无机盐 含量为 0, HPLC 纯度大于 $99.50 \%$, 含量大于 $98.50 \%$.

得到高纯度二甲双胍游离碱后, 本文篮选了二甲双 胍游离碱和硫化氢气体的成盐条件, 最终确定采用硫化 氢丙酮溶液与二甲双胍丙酮溶液滴加沉淀的方法, 制备 了含量大于 $96.00 \%$ 的二甲双胍硫化氢盐.

作者也曾尝试以水为溶剂, 将二甲双胍溶于水中,
再把硫化氢气体直接通入，最后通过冻干获得二甲双胍 硫化氢盐, 所得产品含量 $94.00 \%$. 两种方法相比, 直接 沉淀时间短，同时，目标物从溶液中析出，还可除去溶 解于丙酮的杂质, 所得产物含量高, 且无需在高真空下 长时间冻干获得, 操作简便, 产品质量可控.

\section{2 目标化合物的结构鉴定}

本文通过氢谱、碳谱、元素分析及 $X$ 单晶衍射对二 甲双胍硫化氢盐进行了结构确证.

比较二甲双胍游离碱、盐酸盐和硫化氢盐核磁数据, 大部分信号差别不大, 游离碱的碳谱有一个碳原子的化 学位移和盐酸盐、硫化氢盐有明显差异(161.55 vs 160.09 , 以 $\mathrm{D}_{2} \mathrm{O}$ 为溶剂), 而硫化氢盐的碳谱和盐酸盐无 明显差异 (160.09vs 160.15, 以 $\mathrm{D}_{2} \mathrm{O}$ 为溶剂).

剑桥结构数据中心(The Cambridge Crystallographic Data Centre, CCDC)报道了二甲双胍的盐酸盐、硝酸盐、 醋酸盐以及一些复合物晶体结构, 但未见二甲双胍游离 碱和二甲双胍硫化氢盐的晶体结构报道. 本文培养得到 了二甲双胍游离碱(No. CCDC 1881154)，二甲双胍硫化 氢盐(No. CCDC 1881155)和盐酸盐单晶结构, 如图 1 所 示. 二甲双胍游离碱的亚胺氮原子与另外一个质子化的 亚胺氮上氢原子存在 $\mathrm{N}-\mathrm{H}$ 氢键相互作用.

二甲双胍盐酸盐的单晶结构显示质子化位点在二 甲胺基相邻的亚胺氮原子上，二甲双胍硫化氢盐的单晶 结构也显示出质子化位点在二甲胺基相邻的亚胺氮原 子上，且二甲双胍与硫化氢 $1: 1$ 成盐.

\section{3 目标化合物的含量测定}

目标化合物由硫化氢和二甲双胍组成，所以本文分 别测定其含量.

文献[23]报道硫化氢的测定方法有碘量法、醋酸铅 法和荧光亚甲蓝法, 本文利用此三种方法分别测定二甲 双胍硫化氢盐中硫化氢的含量, 所得结果比较一致(表 1 和表 2), 硫化氢含量均大于 $96.00 \%$. 另外, 通过 HPLC

\begin{tabular}{|c|c|c|c|c|}
\hline \multicolumn{3}{|c|}{ 碘量法 } & \multicolumn{2}{|c|}{ 醋酸铅法 } \\
\hline $\mathrm{m} / \mathrm{g}$ & $V_{\mathrm{Na}_{2} \mathrm{~S}_{2} \mathrm{O}_{3}} / \mathrm{mL}$ & 含量/\% & $\mathrm{m} / \mathrm{g}$ & 含量 $/ \%$ \\
\hline 0.3015 & 13.88 & 96.71 & 0.8162 & 97.70 \\
\hline 0.3014 & 13.91 & 96.87 & 5.1204 & 98.96 \\
\hline
\end{tabular}
测定了二甲双胍的含量, 结果显示其含量大于 $98.50 \%$.

表 1 碘量法和醋酸铅法测定结果

Table 1 Results of iodometry method and lead acetate method

\section{4 二甲双胍硫化氢盐的稳定性研究}

鉴于硫化氢的强还原性，二甲双胍硫化氢盐的稳定 性是其成药性的重要影响因素. 本文分别探索了二甲双 胍硫化氢的溶液稳定性及固体稳定性，结果表明，溶液 
(a)

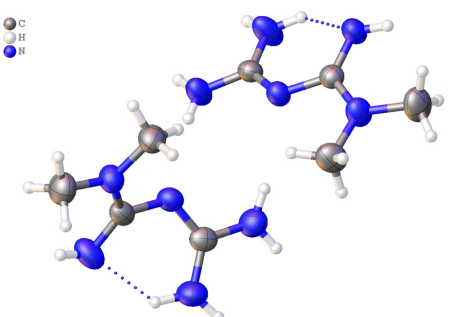

(b)

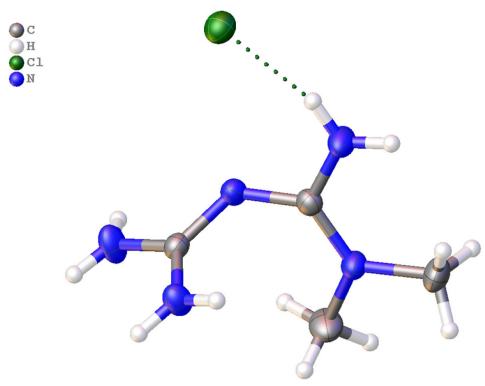

(c)

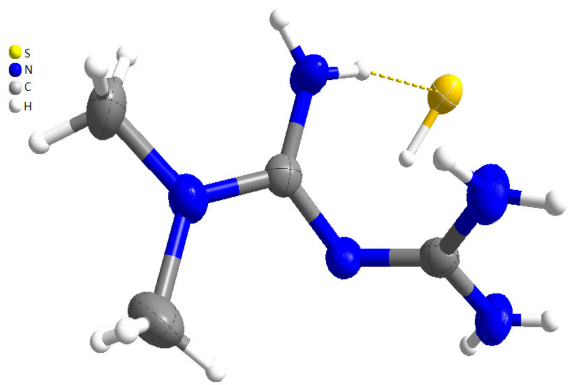

图 1 二甲双胍游离碱(a)、二甲双胍盐酸盐(b)及二甲双胍硫 化氢盐(c)的单晶结构

Figure 1 Crystal structures of metformin (a), metformin hydrochloride (b) and metformin hydrosulfide (c)

表 2 苂光亚甲法测定结果

Table 2 Results of methylene blue spectrophotometric method

\begin{tabular}{lrrr}
\hline \multicolumn{1}{c}{$V / \mathrm{mL}$} & 1.00 & 3.00 & 5.00 \\
\hline 平均值 $A$ & 0.26 & 0.54 & 0.90 \\
$\mathrm{~S}^{2-}$ 含量 $/ \mu \mathrm{g}$ & 10.79 & 28.09 & 50.56 \\
$\mathrm{H}_{2} \mathrm{~S}$ 含量 $/ \%$ & 19.60 & 19.60 & 19.60 \\
含量 $/ \%$ & 98.02 & 98.01 & 98.02 \\
\hline
\end{tabular}

在避光、脱氧、 $4{ }^{\circ} \mathrm{C}$ 情况下, $24 \mathrm{~h}$ 后未见含量明显下降(表 3), 而固体在避光、 $-18{ }^{\circ} \mathrm{C}$ 情况下保存 3 个月也未见含 量明显下降(表 4).

表 3 二甲双胍硫化氢盐溶液稳定性试验

Table 3 Stability of metformin hydrosulfide solution

\begin{tabular}{cccc}
\hline$t / \mathrm{h}$ & $m / \mathrm{g}$ & $V_{\mathrm{Na}_{2} \mathrm{~S}_{2} \mathrm{O}_{3}} / \mathrm{mL}$ & 含量 $/ \%$ \\
\hline 0 & 0.3010 & 14.90 & 96.68 \\
2 & 0.3014 & 14.90 & 96.65 \\
4 & 0.3016 & 14.84 & 96.75 \\
8 & 0.3020 & 14.79 & 96.75 \\
12 & 0.3027 & 14.76 & 96.62 \\
18 & 0.3030 & 14.69 & 96.71 \\
24 & 0.3045 & 14.50 & 96.74 \\
\hline
\end{tabular}

将固体样品密封于西林瓶中, 分别于 1 周、 2 周、1
个月、 3 个月检测含量，结果如表 4 所示, 3 个月内含量 无明显降低(表 4).

表 4 二甲双胍硫化氢盐固体稳定性试验

Table 4 Stability of metformin hydrosulfide

\begin{tabular}{cccc}
\hline$t / \mathrm{d}$ & $m / \mathrm{g}$ & $V_{\mathrm{Na}_{2} \mathrm{~S}_{2} \mathrm{O}_{3}} / \mathrm{mL}$ & 含量 $/ \%$ \\
\hline 7 & 0.3023 & 14.76 & 96.74 \\
15 & 0.3051 & 14.48 & 96.62 \\
30 & 0.3017 & 14.81 & 96.79 \\
90 & 0.3040 & 14.59 & 96.66 \\
\hline
\end{tabular}

\section{2 结论}

本文以盐酸二甲双胍为原料，通过游离得到二甲双 胍, 进而与硫化氢成盐两步反应合成了二甲双胍硫化氢 盐. 其中, 游离二甲双胍的纯度和含量对二甲双胍硫化 氢盐的质量具有决定性影响，本文参考文献采用了金属 钠/乙醇体系、丙酮反复脱盐析晶制得二甲双胍游离碱晶 体, 炽灼残渣结果显示该晶体中没有无机盐的存在, HPLC 纯度大于 $99.50 \%$, 含量大于 $98.50 \%$. 在得到高纯 度的游离二甲双胍后，再通过摸索，确定采用硫化氢丙 酮溶液与二甲双胍丙酮溶液滴加沉淀的方法获得含量 大于 $96.00 \%$ 的二甲双胍硫化氢盐. 含量测定时, 作者比 较了三种方法, 所得结果一致, 相互验证了准确性, 在 后续工作中根据目标物数量的不同可选择不同方法. 如 苂光亚甲蓝样品用量最少, 碘量法适中, 而醋酸铅法虽 操作简便, 用量较大时准确度相对较高, 但大量时, 烘 干至恒重也需要准确判断. 二甲双胍硫化氢盐的溶液及 固体相对稳定，同时其 $0.05 \%$ 浓度水溶液的 $\mathrm{pH}$ 为 8.94 , 上述数据均为二甲双胍硫化氢盐能成为药用硫化氢供 体提供了支撑, 生物学评价工作正在进行中.

\section{3 实验部分}

\section{1 仪器与试剂}

核磁共振仪 (Bruker-AC-E20, Varian-Unity INOVA-400); 高分辨质谱仪(Waters Q-TOF-Premier); 岛津 UV-2450 紫外分光光度计; Varian InovaA400 型核磁共振 仪; X 射线单晶衍射仪(Oxford, Xcalibur EOS); 元素分 析仪(vario MICRO select).

盐酸二甲双胍(苏州亚科科技有限公司). 实验中所 用的试剂除另有说明外，均为市售分析纯. 无水溶剂和 试剂按规定的方法进行干燥处理; 实验用水除另有说明 外, 均指去离子除氧水. 对苯二胺溶液、醋酸锌-醋酸钠 溶液及硫酸铁铵溶液按照文献[24]配制.

\section{2 化合物的合成}

\subsubsection{1,1-二甲基双胍(DMG)游离碱的制备}

在室温下, 向装有机械搅拌、温度计、无水 $\mathrm{CaCl}_{2}$ 
干燥管、温度计及冷凝管的 $3000 \mathrm{~mL}$ 夹套反应瓶中, 加 入无水乙醇 $(1200 \mathrm{~mL})$, 分批加入金属钠(23.45 g, 1.02 $\mathrm{mol}), 20 \mathrm{~min}$ 内加毕, 内温升到 $56{ }^{\circ} \mathrm{C}, 15 \mathrm{~min}$ 后钠块完 全溶解, 然后向反应液中分批加入二甲双胍盐酸盐 (DMGH, $168.90 \mathrm{~g}, 1.02 \mathrm{~mol})$, 内温迅速下降至 $42{ }^{\circ} \mathrm{C}$, 用 $150 \mathrm{~mL}$ 无水乙醇冲洗加料漏斗, 继续剧烈搅拌 $2 \mathrm{~h}$ 后, 得到白色悬浮液. 置冰箱冷至内温 $10{ }^{\circ} \mathrm{C}$ 后, 过滤, 得到澄清滤液, 在 $35{ }^{\circ} \mathrm{C}$ 下减压浓缩, 得到 $124.93 \mathrm{~g}$ 白 色固体粗品 DMG，粗产率 $95 \%$.

\subsubsection{1,1-二甲基双胍游离碱的精制}

室温下, 取上述二甲双胍(DMG)粗品 $60.00 \mathrm{~g}$, 加入 $1500 \mathrm{~mL}$ 无水丙酮, 室温下超声并搅拌直到无大颗粒固 体, 加入 $1.00 \mathrm{~g}$ 活性炭搅拌 $10 \sim 15 \mathrm{~min}$ 过滤. 滤液于 $-18{ }^{\circ} \mathrm{C}$ 析晶 $48 \mathrm{~h}$, 过滤得 $35.72 \mathrm{~g}$ 无色颗粒状晶体 DMG, 产率 $60 \%$. m.p. $118 \sim 120{ }^{\circ} \mathrm{C}$ (lit. ${ }^{[25]}$ m.p. 119 $\left.120{ }^{\circ} \mathrm{C}\right) ;{ }^{1} \mathrm{H}$ NMR $\left(400 \mathrm{MHz}, \mathrm{D}_{2} \mathrm{O}\right) \delta: 2.87(\mathrm{~s}, 6 \mathrm{H}) ;{ }^{13} \mathrm{C}$ NMR (100 MHz, $\left.\mathrm{D}_{2} \mathrm{O}\right) \delta: 37.14$ (2C), 158.44, 161.55.

\subsubsection{1,1-二甲基双胍硫化氢盐(DMGS)的制备}

在已称重的 $500 \mathrm{~mL}$ 三口瓶中, 加入 $250 \mathrm{~mL}$ 丙酮, 室温搅拌下通入 $\mathrm{H}_{2} \mathrm{~S}$ 气体, 通入 $2 \mathrm{~h}$ 后, 称量增重 $5.50 \mathrm{~g}$, 放置待用. 称量 $\mathrm{DMG}$ 的精制品 $(20.83 \mathrm{~g})$ 于 $1000 \mathrm{~mL}$ 雉 形瓶内, 加入 $450 \mathrm{~mL}$ 丙酮, 室温下搅拌直到固体全部 溶解后, 过滤, 将滤液转移到 $1000 \mathrm{~mL}$ 三颈瓶中, 在室 温搅拌下, 用恒压滴液漏斗缓慢滴入上述 $\mathrm{H}_{2} \mathrm{~S}$ 丙酮溶 液, 滴加过程中有白色固体析出. 滴毕, 用 $20 \mathrm{~mL}$ 丙酮 冲洗滴液漏斗, 抽滤, 得白色滤饼, 用 $30 \mathrm{~mL}$ 丙酮洗涤 一次, 真空干燥, 得 $25.35 \mathrm{~g}$ 白色、略有臭味固体 DMGS, 产率 96\%. m.p. 158 $160{ }^{\circ} \mathrm{C} ;{ }^{1} \mathrm{H}$ NMR $\left(400 \mathrm{MHz}, \mathrm{D}_{2} \mathrm{O}\right)$ $\delta: 3.01(\mathrm{~s}, 6 \mathrm{H}) ;{ }^{13} \mathrm{C} \mathrm{NMR}\left(100 \mathrm{MHz}, \mathrm{D}_{2} \mathrm{O}\right) \delta: 37.45(2 \mathrm{C})$, 158.39, 160.15. Anal. calcd for $\mathrm{C}_{4} \mathrm{H}_{13} \mathrm{~N}_{5} \mathrm{~S}$ : C 29.43, H 8.03, N 42.90; found C 29.41, H 7.94, N 42.77. HRMS calcd for $\mathrm{C}_{4} \mathrm{H}_{14} \mathrm{~N}_{5} \mathrm{~S}\left(\mathrm{M}-\mathrm{HS}^{+}\right)$130.1014, found 130.1034 .

\section{3 含量测定}

\subsection{1 碘量法测定硫化氢含量}

参照文献[26]报道的方法, 用碘量法测定二甲双胍 硫化氢盐中硫化物的含量, 结果见表 1 .

\subsection{2 醋酸铅法测定硫化氢含量}

精密称量一定质量的二甲双胍硫化氢盐, 加少量水 溶解后, 再加入过量(4 equiv.)醋酸铅溶液, 室温摚拌 $1 \mathrm{~h}$ 后, 静置冷藏 $30 \mathrm{~min}$, 用精密称量后的玻砂漏斗过滤, 滤饼用水洗涤多次后, $105{ }^{\circ} \mathrm{C}$ 干燥至恒重, 计算二甲双 胍硫化氢的含量, 结果见表 1 .

\subsection{3 荧光亚甲蓝法测定硫化氢含量}

参照文献[24], 配制实验所需的试液及 $\mathrm{Na}_{2} \mathrm{~S}$ 标准溶
液(需临用新制), 进而绘制标准曲线图. 精密称取适量 样品 DMGS，然后按照标准试样的配制方法进行配制并 稀释, 得样品使用液. 分别取 $1.00 、 3.00 、 5.00 \mathrm{~mL}$ 样品 液分别进行 5 次平行试验测定吸光度, 再通过校准曲线 得出硫化物的含量, 通过计算转换为二甲双胍硫化氢盐 的质量分数(见表 2).

\section{3 .4 二甲双胍含量测定}

参考《中国药典》(2015 版)盐酸二甲双胍的含量测 定方法，选择十八烷基硅烷键合硅胶为填充剂的柱子, $1.7 \%$ 磷酸二氢铵溶液 (用磷酸调 $\mathrm{pH}=3.0$ ) 为流动相, 以 $1.0 \mathrm{~min} / \mathrm{mL}$ 的流速, 在波长 $233 \mathrm{~nm}$ 处进行检测. 以二甲 双胍对照品，按外标法以峰面积计算即可得含量.

致谢 感谢四川大学分析测试中心 $\mathrm{X}$ 单晶衍射工作室 罗代兵博士对本文单晶分析的帮助，感谢郑虎教授对本 文的技术指导.

辅助材料(Supporting Information) 盐酸二甲双胍、游 离二甲双胍及二甲双胍硫化氢盐的 ${ }^{1} \mathrm{H}$ NMR、 ${ }^{13} \mathrm{C} \mathrm{NMR}$ 、 HRMS、元素分析、单晶数据与晶体结构图, 以及 HPLC 含量测定的色谱图. 这些材料可以免费从本刊网站 (http://sioc-journal.cn/)上下载.

\section{References}

[1] (a) Zheng, Y.; Yu, B.; De La Cruz, L. K.; Roy Choudhury, M.; Anifowose, A.; Wang, B. H. Med. Res. Rev. 2018, 38, 57.

(b) Guo, W.; Cheng, Z. Y.; Zhu, Y. Z. Acta Pharmacol. Sin. 2013, 34, 1284.

(c) Wallace, J. L.; Wang, R. Nat. Rev. Drug Discovery 2015, 14, 329 .

(d) Zhou, C.; Qiu, B.; Zeng, Y.; Chen, J. P.; Yu, T. J.; Li, Y. Chin. J. Org. Chem. 2017, 37, 92 (in Chinese).

(周婵，邱波，曾毅，陈金平，于天君，李嫕，有机化学，2017，37， 92.)

[2] (a) Hellmich, M. R.; Szabo, C. In Chemistry, Biochemistry and Pharmacology of Hydrogen Sulfide, Vol. 230, Eds.: Moore, P. K.; Whiteman, M., Springer, Switzerland, 2015, p. 233.

(b) Zhao, Y.; Kang, J. M.; Park, C. M.; Bagdon, P. E.; Peng, B.; Xian, M. Org. Lett. 2014, 16, 4536.

(c) Gao, M.; Yu, F. B.; Chen, L. X. Prog. Chem. 2014, 6, 1065 (in Chinese).

(高敏, 于发标, 陈令新, 化学进展, 2014, 6, 1065.)

[3] (a) Sparatore, A.; Santus, G.; Giustarini, D.; Rossi, R.; Del Soldato, P. Expert Rev. Clin. Pharmacol. 2011, 4, 109.

(b) Zhang, X. Z.; Bian, J. S. ACS Chem Neurosci. 2014, 5, 876.

(c) Zhao, Y.; Yang, C. T.; Organ, C.; Li, Z.; Bhushan, S. S.; Otsuka, H.; Pacheco, A.; Kang, J. M.; Aguilar, H. C.; Lefer, D. J.; Xian, M. J. Med. Chem. 2015, 58, 7501.

(d) Kang, J. M.; Li, Z.; Organ, C. L.; Park, C. M.; Yang, C. T.; Pacheco, A.; Wang, D. F.; Lefer, D. J.; Xian, M. J. Am. Chem. Soc. 2016, 138,6336

(e) He, P.; Tang, L. J.; Zhong, K. L.; Hou, S. H.; Yan, X. M. Chin. J. Org. Chem. 2017, 37, 423 (in Chinese).

(何平, 汤立军, 钟克利, 侯淑华, 燕小梅, 有机化学, 2017, 37, 423.)

[4] (a) Benavides, G. A.; Squadrito, G. L.; Mills, R. W.; Patel, H. D.; 
Isbell, T. S.; Patel, R. P.; DarleyUsmar, V. M.; Doeller, J. E.; Kraus, D. W. Proc. Natl. Acad. Sci. 2007, 104, 17977.

(b) Liang, D.; Wu, H.; Wong, M. W.; Huang, D. Org. Lett. 2015, 17, 4196.

[5] (a) Zhao, Y.; Biggs, T. D.; Xian, M. Chem. Commun. (Camb.) 2014, 50,11788 .

(b) Martelli, A.; Testai, L.; Citi, V.; Marino, A.; Pugliesi, I.; Barresi, E.; Nesi, G.; Rapposelli, S.; Taliani, S.; Da Settimo, F.; Breschi, M. C.; Calderone, V. ACS Med. Chem. Lett. 2013, 4, 904.

[6] (a) Martelli, A.; Testai, L.; Citi, V.; Marino, A.; Bellagambi, F. G.; Ghimenti, S.; Breschi, M. C.; Calderone, V. Vasc. Pharmacol. 2014, $60,32$.

(b) Citi, V.; Martelli, A.; Testai, L.; Marino, A.; Breschi, M. C.; Calderone, V. Planta Med. 2014, 80, 610.

[7] Zhao, Y.; Wang, H.; Xian, M. J. Am. Chem. Soc. 2011, 133, 15

[8] Ozturk, T.; Ertas, E.; Mert, O. Chem. Rev. 2007, 107, 5210.

[9] (a) Qandil, A. M. Int. J. Mol. Sci. 2012, 13, 17244.

(b) Li, L.; Rossoni, G.; Sparatore, A.; Lee, L. C.; Del Soldato, P.; Moore, P. K. Free Radicals Biol. Med. 2007, 42, 706.

[10] (a) Wallace, J. L.; Caliendo, G.; Santagada, V.; Cirino, G.; Fiorucci, S. Gastroenterology 2007, 132, 261.

(b) Chattopadhyay, M.; Kodela, R.; Nath, N.; Dastagirzada, Y. M.; Velazquez-Martinez, C. A.; Boring, D.; Kashfi, K. Biochem. Pharmacol. 2012, 83, 715 .

[11] Lee, Z. W.; Zhou, J.; Chen, C. S.; Zhao, Y.; Tan, C. H.; Li, L.; Moore, P. K.; Deng, L. W. PLoS One 2011, 6, e21077.

[12] Devarie-Baez, N. O.; Bagdon, P. E.; Peng, B.; Zhao, Y.; Park, C.-M.; Xian, M. Org. Lett. 2013, 15, 2786.

[13] Fukushima, N.; Ieda, N.; Sasakura, K.; Nagano, T.; Hanaoka, K.; Suzuki, T.; Miyata, N.; Nakagawa, H. Chem. Commun. (Camb.) 2014, 50, 587

[14] Fukushima, N.; Ieda, N.; Kawaguchi, M.; Sasakura, K.; Nagano, T.; Hanaoka, K.; Miyata, N.; Nakagawa, H. Bioorg. Med. Chem. Lett.
2015, 25, 175.

[15] Zhou, Z.; von Wantoch Rekowski, M.; Coletta, C.; Szabo, C.; Bucci, M.; Cirino, G.; Topouzis, S.; Papapetropoulos, A.; Giannis, A. Bioorg. Med. Chem. 2012, 20, 2675.

[16] Zheng, Y.; Yu, B.; Ji, K.; Pan, Z.; Chittavong, V.; Wang, B. Angew. Chem., Int. Ed. 2016, 55, 4514.

[17] Steiger, A. K.; Pardue, S.; Kevil, C. G.; Pluth, M. D. J. Am. Chem. Soc. 2016, 138, 7256.

[18] Zheng, H.; Weng, L. L. CN 201710475428, 2017.

[19] Achterhof, M.; Conaway, R. F.; Boord, C. E. J. Am. Chem. Soc. 1931, 53, 2682.

[20] Romero, R.; Erez, O.; Huttemann, M.; Maymon, E.; Panaitescu, B.; Conde-Agudelo, A.; Pacora, P.; Yoon, B. H.; Grossman, L. I. Am. J. Obstet. Gynecol. 2017, 217, 282.

[21] Ye, Y.; Li, X. J.; Zeng, Z. Z. Chin. J. Appl. Chem. 2005, 22, 1060 (in Chinese).

(叶琰, 李秀娟, 曾正志, 应用化学, 2005, 22, 1060.)

[22] (a) Moghimi, A.; Khavassi, H.; Dashtestani, F.; Kordestani, D.; Jafari, A. E.; Maddah, B.; Moosavi, S. J. Mol. Struct. 2011, 996, 38. (b) Veisi, H.; Masti, R.; Kordestani, D.; Safaei, M.; Sahin, O. J. Mol. Catal. A: Chem. 2014, 385, 61.

(c) Alizadeh, A.; Khodaei, M. M.; Abdi, G.; Kordestani, D. Bull. Korean Chem. Soc. 2012, 33, 3640.

[23] Zhang, Z. L.; Bai, B.; Song, H. Chem. Eng. 2012, 26, 34 (in Chinese). (张子龙，白冰，宋华，化学工程师, 2012，26, 34.)

[24] Guan, X. C.; Peng, H. Y. J. Anyang Inst. Technol. 2012, 11, 25 (in Chinese).

(关秀存, 彭红艳, 安阳工学院学报, 2012, 11, 25.)

[25] Koh, M.; Lee, J.-C.; Min, C.; Moon, A. Bioorg. Med. Chem. 2013, 21, 2305.

[26] Zou, J. Y.; Feng, J. T. Sulphuric Acid Ind. 2013, 38 (in Chinese). (邹婕妤，冯俊婷，硫酸工业，2013，38.) 